\title{
miR-214-3p promotes the proliferation, migration and invasion of osteosarcoma cells by targeting CADM1
}

\author{
HAIQING CAI, MINGYUAN MIAO and ZHIGANG WANG
}

\author{
Pediatric Orthopedic Department, Shanghai Children's Medical Center, \\ Shanghai Jiaotong University School of Medicine, Shanghai 200031, P.R. China
}

Received April 22, 2017; Accepted February 27, 2018

DOI: $10.3892 / \mathrm{ol} .2018 .8927$

\begin{abstract}
Although osteosarcoma (OS) is the most common type of primary bone tumor in adolescents and young adults, its mechanism remains unclear. A previous study by the authors demonstrated that miR-214-3p was upregulated in OS patients. Therefore, the present study aimed to investigate the effect and molecular mechanism of miR-214-3p in OS cells. OS cell lines, U2OS and MNNG/HOS $\mathrm{Cl}$ 5, were transiently transfected with miR-214-3p mimics, a control mimic, miR-214-3p inhibitors and a control inhibitor. Subsequent assays revealed that elevated miR-214-3p promoted the proliferative, migratory and invasive abilities of OS cells, while the opposite effects were observed in cells that were transfected with miR-214-3p inhibitors. The interaction between miR-214-3p and cell adhesion molecule 1 (CADM1) 3'untranslated region (UTR) was verified by a dual luciferase assay, which indicated that the relative luciferase activity was decreased in $293 \mathrm{~T}$ cells that were co-transfected with miR-214-3p mimic and psiCHECK2-CADM1-3'UTR compared with cells that were co-transfected with psiCHECK2-CADM1-3'UTR and control mimic. The knockdown of CADM1 using small-interfering RNA enhanced the proliferative, migratory and invasive abilities of OS cells. Furthermore, downregulated CADM1 expression increased the expression of phosphorylated P44/42 mitogen activated kinase (MAPK). In conclusion, miR-214-3p was able to directly target CADM1 and decrease its expression. This resulted in the activation of the P44/42 MAPK signaling pathway, and thereby promoted the proliferation, migration and invasion of OS cells.
\end{abstract}

Correspondence to: Dr Zhigang Wang, Pediatric Orthopedic Department, Shanghai Children's Medical Center, Shanghai Jiaotong University School of Medicine, 1678 Dongfang Road, Pudong, Shanghai 200031, P.R. China

E-mail: gangqing@vip.sina.com

Key words: microRNA-214-3p, osteosarcoma, cell adhesion molecule 1, P44/42 mitogen activated kinase, proliferation, migration, invasion

\section{Introduction}

Osteosarcoma (OS) is the most common type of primary bone tumor in adolescents and young adults with an incidence of 4-5/1,000,000 and a 5-year overall survival rate of $60-70 \%$ (1-3). A number of factors have been demonstrated to affect the prognosis, including the axial localization of the primary tumor, the tumor diameter and the histological response to preoperative chemotherapy (4). Although extensive advancements in diagnostic methods and surgical techniques have been developed, the molecular etiology of osteosarcoma has not been fully elucidated (5).

MicroRNAs (miRNAs/miRs) are small non-coding RNAs of 20-25 nucleotides, which have been demonstrated to function in various biological processes, including cancer initiation, growth and progression, by targeting genes for post-transcriptional degradation via their 3'untranslated region (UTR) $(6,7)$.

miR-214, located within the sequence of the long non-coding Dmn3os transcript, has been reported not only to be highly dysregulated but also highly variable in its expression level in multiple types of cancer $(8,9)$. This suggests that miR-214 may function as a tumor suppressor and may have a tumorigenic role. In previous studies, researchers have demonstrated that miR-214 was upregulated in osteosarcoma and associated with tumor progression and poor prognosis $(10,11)$. However, little progress has been achieved in terms of elucidating the molecular mechanisms of miR-214-3p-mediated tumorigenesis in OS.

In the present study, it was demonstrated that miR-214-3p directly targeted the 3'-UTR of cell adhesion molecule 1 (CADM1) and therefore suppressing the expression of CADM1. The transfection of miR-214-3p mimic was able to promote the proliferation, migration and invasion of OS cells in vitro by activating the P44/42 mitogen activated kinase (MAPK) signaling pathway.

\section{Materials and methods}

Cell culture. Human OS cell lines, MNNG/HOS Cl\#5 and U2OS, and normal human 293T cells were obtained from The Cell Bank of Type Culture Collection of Chinese Academy of Sciences (Shanghai, China) and cultured in Dulbecco's modified Eagle's medium (DMEM), supplemented with $10 \%$ fetal bovine serum (FBS) and $100 \mathrm{U} / \mathrm{ml}$ penicillin-streptomycin 
(all from HyClone; GE Healthcare, Chicago, IL, USA). The cells were cultured at $37^{\circ} \mathrm{C}$ in a humidified incubator containing $5 \% \mathrm{CO}_{2}$.

RNA isolation and reverse transcription-quantitative polymerase chain reaction ( $R T-q P C R)$. Total RNA of MNNG/HOS $\mathrm{Cl}$ 5 and U2OS was isolated using TRIzol (Thermo Fisher Scientific, Inc., Waltham, MA, USA), according to the manufacturer's protocol. Reverse transcription of mature miRNAs was performed using $1 \mu \mathrm{g}$ RNA, M-MLV Reverse Transcriptase (catalog no. M1701), Recombinant RNasin ${ }^{\circledR}$ Ribonuclease inhibitor (catalog no. N2511) and dNTP (catalog. no U1515) (all from Promega Corporation, Madison, WI, USA) as described in a previous study (12). The stem-loop primer sequences used are as follows: miR-214-3p, 5'-CTCAACTGG TGTCGTGGAGTCGGCAATTCAGTTGAGACTGCCTG-3' and U6, 5'-CGCTTCACGAATTTGCGTGTCAT-3'. Reverse transcription of CADM1 was performed using the ReverTra Ace ${ }^{\circledR}$ PCR-qRT kit (catalog no. FSQ-101; Toyobo Life Science, Osaka, Japan) according to the manufacturer's protocol.

The expression of CADM1 mRNA and mature miR-214-3p was determined by RT-qPCR using a KAPA SYBR ${ }^{\circledR}$ FAST Universal qPCR kit (catalog no. KK4601; Kapa Biosystems, Inc., Wilmington, MA, USA), according to the manufacturer's protocol. In brief, $20 \mu \mathrm{l}$ mixture was heated at $95^{\circ} \mathrm{C}$ for $3 \mathrm{~min}$ for enzyme activation, then the $20 \mu \mathrm{l}$ reaction mixture were incubated as follows: $95^{\circ} \mathrm{C}$ for $3 \mathrm{sec}$ and $60^{\circ} \mathrm{C}$ for $20 \mathrm{sec}$ for 40 cycles. $18 \mathrm{~S}$ and U6 were used as internal controls for CADM1 and miR-214-3p, respectively. The primer sequences (Beijing Genomics Institute, Shenzhen, Guangdong, China) used are as follows: CADM1, forward, 5'-GCAAATCGGAGG TGGAAGA-3', and reverse, 5'-GCACTTGAGGCTTATACT GTACTT-3'; 18S, forward, 5'-CAGCCACCCGAGATTGAG CA-3', and reverse, 5'-TAGTAGCGACGGGCGGTGTG-3'; miR-214-3p, forward, 5'-ACACTCCAGCTGGGACAGCAG GCACAGACA-3', and reverse, 5'-TGGTGTCGTGGAGTC G-3', and U6, forward, 5'-CTCGCTTCGGCAGCACATATA CT-3', and reverse, 5'-ACGCTTCACGAATTTGCGTGTC-3'. The $2^{-\Delta \Delta \mathrm{Cq}}$ method was used to quantify the expression of miR-214-3p and CADM1, and each experiment was performed in triplicate (13).

Cell transfection. A total of $2 \times 10^{5} \mathrm{MNNG} / \mathrm{HOS} \mathrm{Cl \# 5}$ or U2OS cells were seeded per well into 6 -well plates and transfected transiently with $50 \mathrm{nM}$ miR-214-3p mimic/inhibitor and NC/inhibitor NC (Shanghai GenePharma Co., Ltd., Shanghai, China) or CADM1 siRNA and siNC (Biotend, Shanghai, China) using $3 \mu$ l Lipofectamine ${ }^{\circledR} 2000$ (Thermo Fisher Scientific, Inc.) according to the manufacturer's protocol. The sequences of miRNA mimics, inhibitor, NC and CAM1 siRNAs 1-3 used in assays are presented in Table I. During transfection, DMEM without FBS was used. For the cell function assay, cells were collected $12 \mathrm{~h}$ after transfection. For RT-qPCR and western blotting, cells were collected 24 and $48 \mathrm{~h}$ after transfection. For the dual luciferase assay, cells were collected $48 \mathrm{~h}$ after transfection.

MTT assay. Transfected MNNG/HOS Cl\#5 or U2OS cells were seeded into 96 -well plates at $3 \times 10^{3}$ cells/well. A total of $20 \mu \mathrm{l}$ MTT (5 mg/ml; Sangon Biotech Co., Ltd., Shanghai,
China) was added to each well, and incubated with the cells for $4 \mathrm{~h}$ at $37^{\circ} \mathrm{C}$. Supernatant fractions were discarded, and $150 \mu \mathrm{l}$ dimethyl sulfoxide was added to each well to dissolve the crystals. Absorbance values were obtained at $490 \mathrm{~nm}$ in quintuplicate using a spectrophotometric plate reader (Infinite ${ }^{\circledR} 200$ PRO; Tecan Group, Ltd., Mannedorf, Switzerland).

Scratch assay. The transfected MNNG/HOS Cl\#5 or U2OS cells were seeded into 12-well plates to form adherent monolayers. A 200- $\mu$ l pipette tip was used to make a scratch, and then the plates were washed twice in PBS to remove the resultant debris and floating cells. The cell culture medium was immediately replaced with DMEM containing $1 \%$ FBS. The images of the scratch were taken at different time points $(0,12,24$ and 48 h) using a Leica Microsystems DMI3000B light microscope (Leica Microsystems GmbH, Wetzlar, Germany). The assays were performed in triplicate.

Transwell assay. For the migration assay, transfected cells were suspended in FBS-free DMEM medium containing $0.1 \%$ bovine serum albumin (Bioworld Technology, Inc., St. Louis Park, MN, USA) and $5 \times 10^{4}$ cells $/ 200 \mu$ l were seeded into the upper chamber. For the invasion assay, Matrigel (BD Biosciences, Franklin Lakes, NJ, USA) was diluted 1:4 with serum-free DMEM medium and used to coat the Transwell inserts (pore size, $8-\mu \mathrm{m}$; EMD Millipore, Billerica, MA, USA) to form a matrix barrier. Transfected cells were suspended in FBS-free DMEM medium containing $0.1 \%$ bovine serum albumin, and $5 \times 10^{4}$ cells $/ 200 \mu \mathrm{l}$ were seeded into the upper chamber. A total of $600 \mu \mathrm{l}$ medium containing $15 \%$ FBS was added to the lower chamber. The cells were incubated at $37^{\circ} \mathrm{C}$ for different durations. Then, the cells that had migrated or invaded through the membrane were fixed with $95 \%$ ethyl alcohol for $15 \mathrm{~min}$ at room temperature and stained with $0.1 \%$ crystal violet for $10 \mathrm{~min}$ at room temperature. The images of the cells on the lower surface were captured and counted in 5 random fields of view.

Dual luciferase assay. The human CADM1 3'UTR seed region was amplified by PCR using the following primers: CADM1 3'UTR forward, 5'-GGCCTCGAGGGAACTTGCGAGAAA TTCGTGT-3', and reverse, 5'-TTAAGCGGCCGCAATGCG AATGGGAACATATGGA-3'. The transcript was then cloned into a psiCHECK-2 vector (Promega Corporation), downstream of the Renilla luciferase gene. The vector also contained the Firefly luciferase gene. A total of $4 \times 10^{5} 293 \mathrm{~T}$ cells were seeded per well into 6-well plates and co-transfected with either $50 \mathrm{nM}$ miR-214-3p mimics or miRNA NC and $2 \mu \mathrm{g}$ plasmid vector using Lipofectamine ${ }^{\circledR} 2000$, according to the manufacturer's protocol. The cells were lysed and assayed for luciferase activity at $48 \mathrm{~h}$ post-transfection using a Dual-Luciferase Assay kit (catalog no. E1910; Promega Corporation). The assays were independently repeated $\geq 3$ times. The firefly luciferase was used as a reference for normalization.

Western blotting. The cells were lysed using radioimmunoprecipitation assay buffer (catalog no. P00138; Beyotime Institute of Biotechnology, Haimen, China) supplemented with protease inhibitors (Complete ${ }^{\mathrm{TM}}$ Protease Inhibitor Cocktail; catalog no. 04693116001; Roche Diagnostics, 
Table I. Sequences of miRNA mimics, inhibitor, NC and CAM1 siRNAs 1-3.

\begin{tabular}{lll}
\hline Name & Sense/antisense & \multicolumn{1}{c}{ Sequence } \\
\hline miR-214-3p mimics & Sense & 5'-ACAGCAGGCACAGACAGGCAGU-3' \\
& Antisense & 5'-UGCCUGUCUGUGCCUGCUGUUU-3' \\
miRNA NC & Sense & 5'-UUCUCCGAACGUGUCACGUTT-3' \\
miR-214 inhibitor & Antisense & 5'-ACGUGACACGUUCGGAGAATT-3' \\
miRNA inhibitor NC & & 5'-ACUGCCUGUCUGUGCCUGCUGU-3' \\
CADM1-siRNA-1 & & 5'-CAGUACUUUUGUGUAGUACAA-3' \\
& Sense & 5'-GGUGGAAGGUGAGGAGAUUdTdT-3' \\
CADM1-siRNA-2 & Antisense & 5'-AAUCUCCUCACCUUCCACCdTdT-3' \\
& Sense & 5'-UCAGGUGGUUCAAAGGGAAdTdT-3' \\
CADM1-siRNA-3 & Antisense & 5'-UUCCCUUUGAACCACCUGAdTdT-3' \\
& Sense & 5'-CCAACCUGUUCAUCAAUAAdTdT-3' \\
siNC & Antisense & 5'-UUAUUGAUGAACAGGUUGGdTdT-3' \\
& Sense & 5'-UUCUCCGAACGUGUCACGUdTdT-3' \\
& Antisense & 5'-ACGUGACACGUUCGGAGAAdTdT-3'
\end{tabular}

miRNA, microRNA; siRNA, small interfering RNA; NC, negative control; CADM1, cell adhesion molecule 1.

Basel, Switzerland; PMSF; catalog no. ST505; Beyotime Institute of Biotechnology) and phosphatase inhibitors

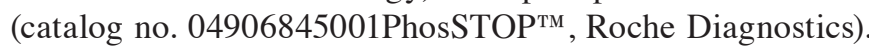
The supernatant was collected, and the protein concentration was quantified using a BCA kit and a plate reader (Infinite ${ }^{\circledR}$ M200 PRO, Group, Ltd., Mannedorf, Switzerland). A total of $30 \mu \mathrm{g}$ protein was subjected to $10 \%$ SDS-PAGE and transferred to polyvinylidene fluoride membranes for $120 \mathrm{~min}$ at $200 \mathrm{~mA}$. Then, the blots were blocked with $5 \%$ fat free milk at room temperature for $1 \mathrm{~h}$ and incubated overnight at $4^{\circ} \mathrm{C}$ with anti-P44/42 MAPK (dilution, 1:1,000; catalog no. 4695; Cell Signaling Technology, Inc., Danvers, MA, USA), anti-phospho-P44/42 MAPK (dilution, 1:1,000; catalog no. 4370; Cell Signaling Technology), anti-CADM1 (dilution, 1:1,000; catalog no. 14335-1-AP, ProteinTech Group, Inc., Chicago, IL, USA) and anti-GAPDH (AP0063, Bioworld Technology, Inc., St. Louis Park, MN, USA). Following washing three times with PBST (1xPBS with $1 \%$ Tween-20) for $5 \mathrm{~min}$, the blots was incubated with horseradish peroxidase-conjugated goat anti-rabbit (dilution, 1:5,000; catalog no. 4412; Cell Signaling Technology) at room temperature for $1 \mathrm{~h}$, and then washed with PBST for $5 \mathrm{~min}$ three times. The proteins were visualized using Pierce ECL Western Blotting substrate (catalog no. 32209; Invitrogen; Thermo Fisher Scientific, Inc.) and a Tanon 5200 Multi system (Tanon Science and Technology Co., Ltd., Shanghai, China). The grayscale value was measured using ImageJ software (version no. 2006.02.01; National Institutes of Health, Bethesda, MD, USA).

Bioinformatics analysis. Potential target genes of miR-214-3p were predicted using TargetScan Human 7.1 (http://www. targetscan.org/vert_71/) and mirTarBase (http://mirtarbase. mbc.nctu.edu.tw/php/index.php), and miR-214-3p was used as a search term. The Kyoto Encyclopedia of Genes and Genomes pathways of the potential target genes were predicted using
GeneCoDis (http://genecodis.cnb.csic.es/) and the target genes of miR-214-3p were used as search terms. All the sites were accessed on May 20th, 2015.

Statistical analysis. All statistical analyses were performed using SPSS (version 21.0; IBM Corp., Armonk, NY, USA). Comparisons between 2 different groups were performed using Student's t-test. Comparisons between $\geq 3$ independent groups were performed using one-way analysis of variance (ANOVA) followed by Scheffé's post hoc test was used. Multivariate ANOVA was used for the comparison of multiple groups at different time points. All data are presented as the mean \pm standard deviation. $\mathrm{P}<0.05$ was considered to indicate a statistically significant difference.

\section{Results}

miR-214-3p affects the proliferation, migration and invasion of OS cells. In a previous study by the authors, miR-214-3p expression was detected in cancerous and noncancerous bone tissues from 92 children treated for primary osteosarcoma (12). The previous study revealed that upregulated expression of miR-214 may be associated with tumor progression and adverse prognosis in pediatric osteosarcoma $(13,14)$. To investigate the effect of miR-214-3p on OS in vitro, miR-214-3p mimics and miR-NC were transfected into U2OS and MNNG/HOS $\mathrm{Cl} \# 5$ cells in the present study (Fig. 1A). A MTT assay was performed to examine the proliferative ability of OS cells that overexpress miR-214-3p. Upregulated miR-214-3p expression was able to significantly increase the proliferation of OS cells (Fig. 1B). To evaluate the role of miR-214-3p in migration and invasion, scratch assay and Transwell assays were performed, revealing that miR-214-3p mimic was able to promote the migration and invasion of OS cells (Fig. 1C-E). In addition, inhibition of miR-214-3p expression in OS cells reduced their proliferative, migratory and invasive abilities compared with 

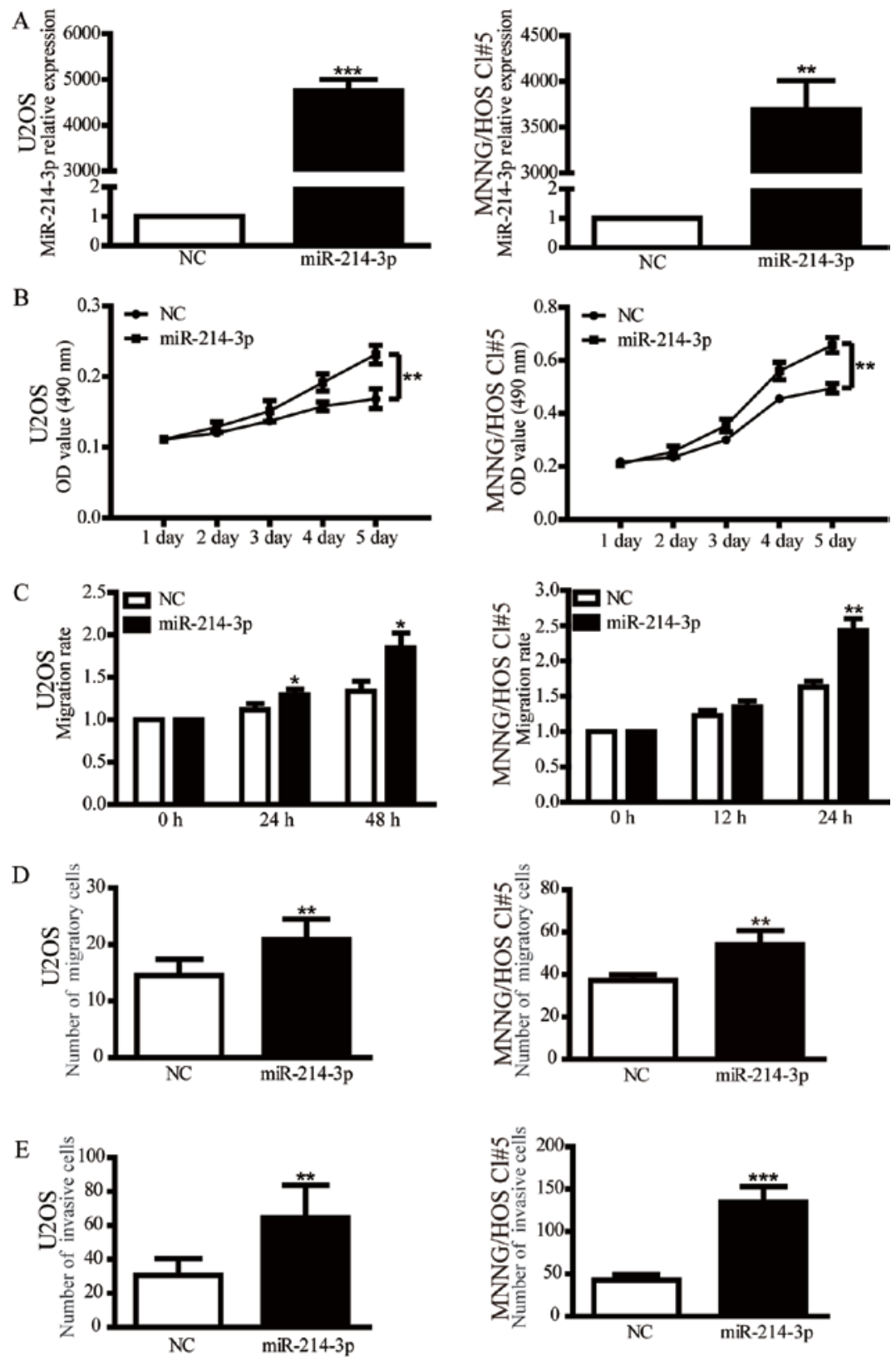

Figure 1. Upregulated miR-214-3p expression promotes proliferation, migration and invasion of osteosarcoma cells. (A) Relative expression of miR-214-3p in U2OS and MNNG/HOS Cl\#5 cells that were transiently transfected with miR-214-3p or NC. U6 was used as an internal control. The y-axis refers to the fold change. The value of each sample was calculated using the $2^{-\triangle \Delta \mathrm{Cq}}$ method and analyzed using Student's t-test. Data are presented as the mean \pm standard deviation. (B) Growth curves of cells that were transfected with miR-214-3p or NC as detected by MTT assays. The OD values were detected at $490 \mathrm{~nm}$. (C) A scratch assay was used to detect the motility of U2OS and MNNG/HOS Cl\#5 cells that were transfected with miR-214-3p or NC. Migration rate is expressed as fold change relative to NC and miR-214-3p at $0 \mathrm{~h}$. (D) Quantitative results of Transwell migration assays in miR-214-3p-overexpressing U2OS and MNNG/HOS Cl\#5 cells. U2OS cells and MNNG/HOS Cl\#5 cells were incubated for 24 and $12 \mathrm{~h}$, respectively (E) Quantitative results of the Transwell invasion assay in miR-214-3p-overexpressing U2OS and MNNG/HOS Cl\#5 cells. U2OS cells and MNNG/HOS Cl\#5 cells were incubated for 48 and $24 \mathrm{~h}$, respectively. ${ }^{*} \mathrm{P}<0.05,{ }^{* *} \mathrm{P}<0.01$ and ${ }^{* * * *} \mathrm{P}<0.001$ vs. NC. miR, microRNA; NC, negative control; OD, optical density.

the inhibitor NC group (Fig. 2). These findings indicate that miR-214-3p may act as an oncogene in OS cells.

CADM1 is a direct target of $m i R-214-3 p$. To elucidate the underlying molecular mechanisms of miR-214-3p in the proliferation, migration and invasion of OS cells, the potential target genes of miR-214-3p were predicted using TargetScan Human 7.1 and mirTarBase. The pathways of the potential target genes were predicted using GeneCoDis (Fig. 3A). CADM1 was identified as a putative target of miR-214-3p, and the potential binding site between miR-214-3p and CADM1 is presented in Fig. 3B. CADM1 was selected for further validation by examining CADM1 mRNA expression following overexpression or knockdown of miR-214-3p in U2OS and MNNG/HOS Cl\#5 cells. CADM1 expression was suppressed by overexpression of miR-214-3p and increased by knockdown of miR-214-3p (Fig. 3C). To further verify whether miR-214-3p targets CADM1 directly, the CADM1 3'UTR was cloned into psiCHECK-2 prior to dual luciferase assay. As indicated in Fig. 3D, a significant decrease in the Renilla luciferase/Firefly luciferase ratio was identified following co-transfection of the CADM1-3'UTR plasmid with miR-214-3p mimic, but not with 

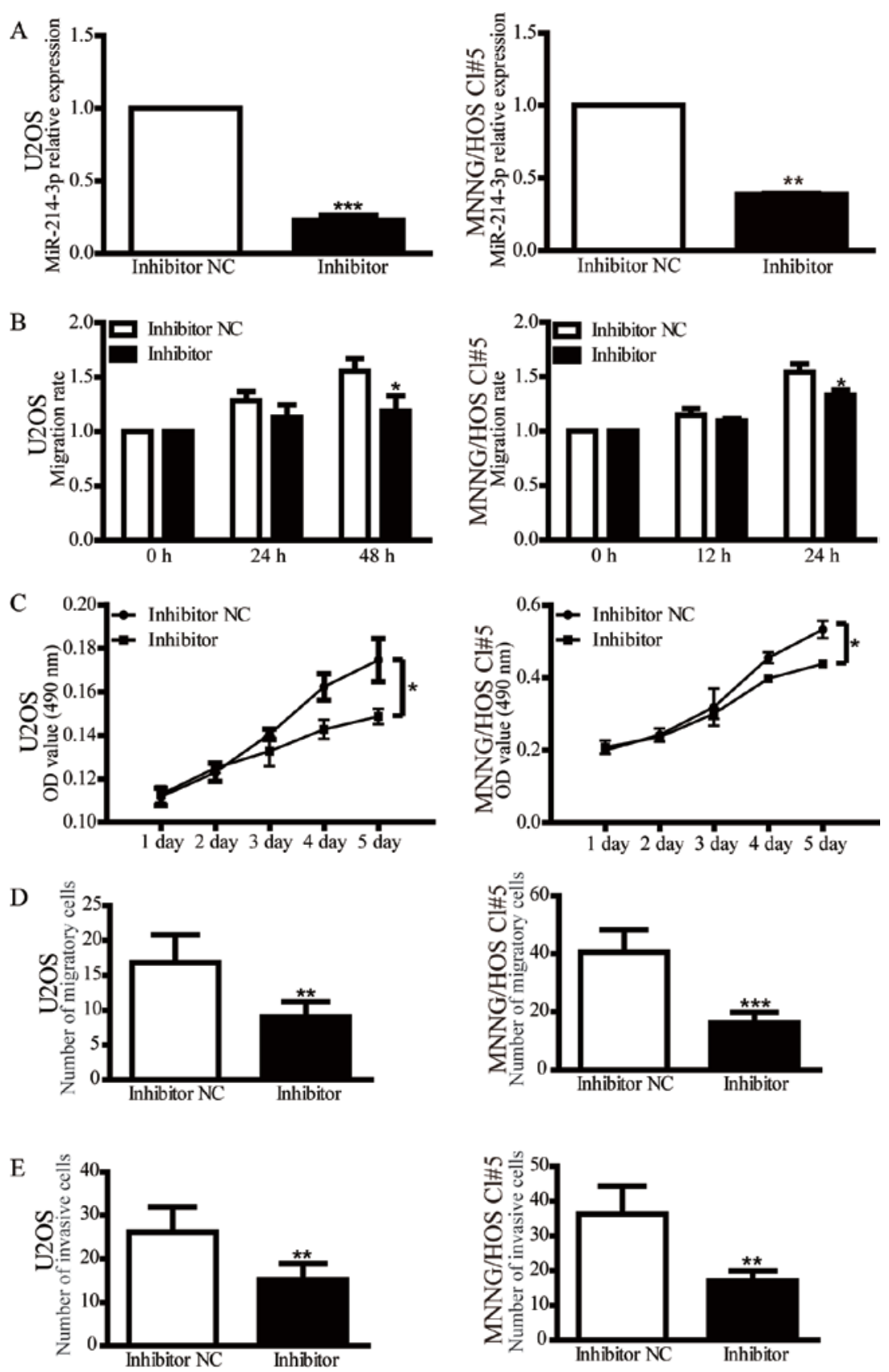

Figure 2. Knockdown of miR-214-3p inhibits proliferation, migration and invasion in osteosarcoma cells. (A) Relative expression of miR-214-3p in U2OS and MNNG/HOS Cl\#5 cells that were transiently transfected with miR-214-3p inhibitors or inhibitor NC. U6 was used as an internal control. The value of each sample was calculated using the $2^{-\Delta \Delta \mathrm{Cq}}$ method and analyzed using Student's t-test. Data are presented as the mean \pm standard deviation. (B) Scratch assays were used to detect the motility of U2OS and MNNG/HOS Cl\#5 cells that were transfected with miR-214-3p inhibitor or inhibitor NC. Migration rate is expressed as fold change relative to inhibitor NC and miR-214-3p inhibitor at $0 \mathrm{~h}$. (C) Growth curves of osteosarcoma cells that were transfected with miR-214-3p inhibitor or inhibitor NC as detected by MTT assays. The OD values were detected at $490 \mathrm{~nm}$. (D) Quantitative results of Transwell migration assays in miR-214-3p-downregulated U2OS and MNNG/HOS Cl\#5 cells. U2OS and MNNG/HOS Cl\#5 cells were incubated for 24 and $12 \mathrm{~h}$, respectively. (E) Quantitative results of Transwell invasion assay in miR-214-3p-downregulated U2OS and MNNG/HOS Cl\#5 cells. U2OS and MNNG/HOS Cl\#5 cells were incubated for 48 and $24 \mathrm{~h}$, respectively. ${ }^{*} \mathrm{P}<0.05,{ }^{* * *} \mathrm{P}<0.01$ and ${ }^{* * * *} \mathrm{P}<0.001$ vs. inhibitor NC. miR, microRNA; NC, negative control; OD, optical density.

miR-NC, suggesting that miR-214-3p may be able to repress CADM1 expression by directly binding the 3'UTR of CADM1.

CADM1 knockdown facilitates the proliferation, migration and invasion of $O S$ cells by activating P44/42 signaling. To further verify the effect of CADM1 on OS cells, U2OS and MNNG/HOS Cl\#5 cells were separately transfected with 3 CADM1 siRNAs (siCADM1s). siCADM1-3 was selected for subsequent experiments due to its higher interference efficiency
(Fig. 4A). The assays demonstrated that the knockdown of CADM1, similarly to the overexpression of miR-214-3p, was able to promote proliferation, migration and invasion of OS cells (Fig. 4B-E).

In order to elucidate the mechanism of CADM1 in regulating the growth and motility of OS cells, western blotting was used to identify the signaling pathway implicated in the process. The level of phosphorylated P44/42 MAPK was elevated in CADM1-knocked down cells compared with 
A

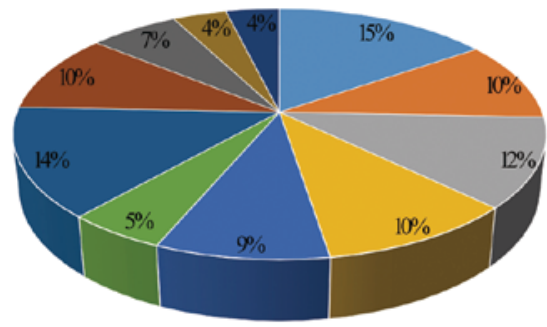

[1 Regulation of actin cytoskeleton

n Ubiquitin mediated proteolysis

ㅁ. Calcium signaling pathway

nnt signaling pathway

- Cell adhesion molecules (CAMs)

- Fe gamma R-mediated phagocytosis, regulation of actin cytoskeleton

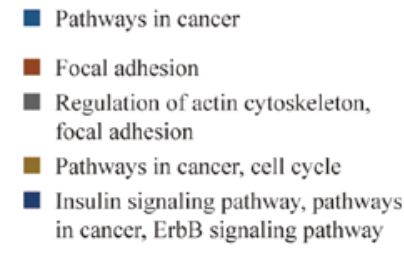

Focal adhesion

Regulation of actin cytoskeleton, focal adhesion

- Insulin signaling pathway, pathways in cancer, ErbB signaling pathway

B
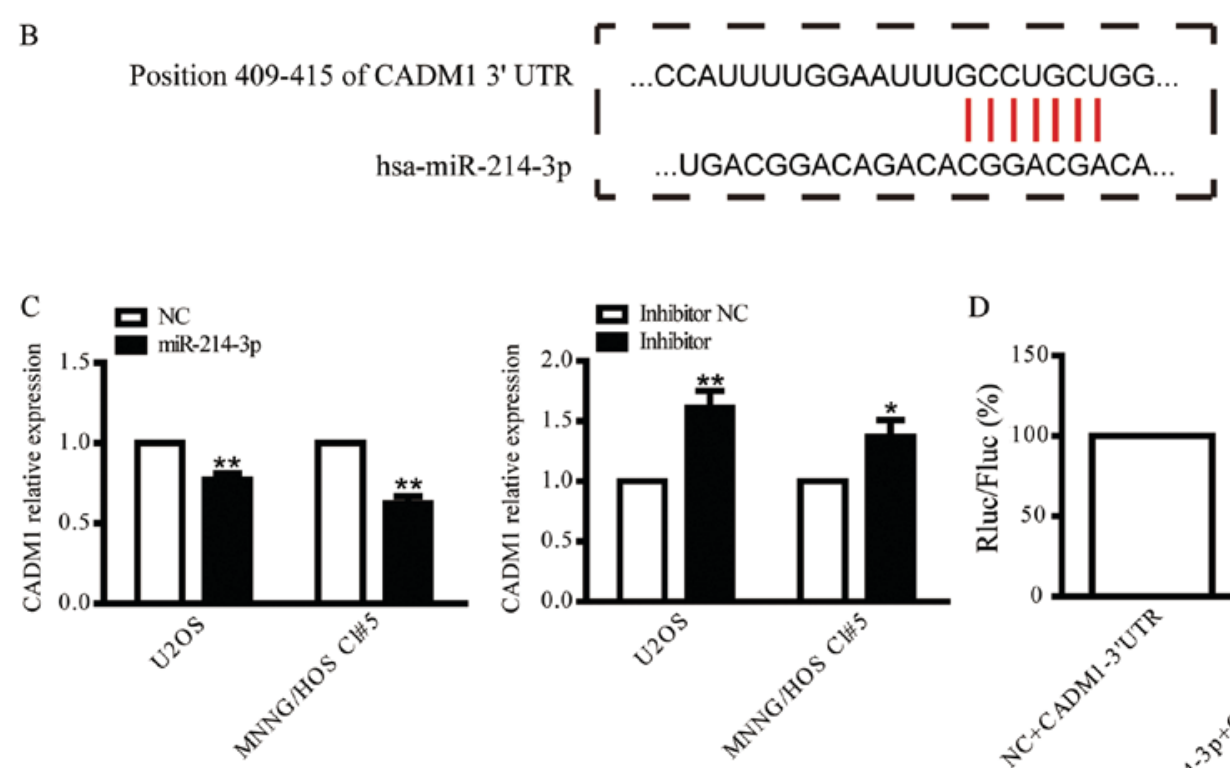

D

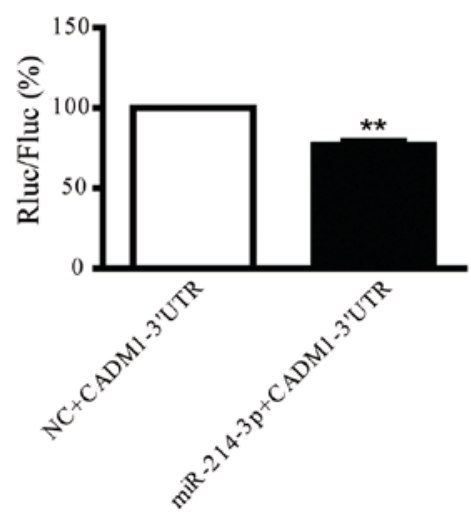

Figure 3. miR-214-3p directly targets the 3'UTR of CADM1. (A) Kyoto Encyclopedia of Genes and Genomes pathways that are associated with tumor characteristics were predicted by GeneCodis. (B) The binding site between miR-214-3p and CADM1 3'UTR as predicted by TargetScan. (C) CADM1 expression in $\mathrm{U} 2 \mathrm{OS}$ and MNNG/HOS Cl\#5 cells that were transiently transfected with miR-214-3p or inhibitor, relative to NC. 18S was used as an internal control. The value of each sample was calculated using the $2^{-\Delta \Delta C q}$ method and analyzed using Student's t-test. Data are presented as the mean \pm standard deviation. (D) $293 \mathrm{~T}$ cells were co-transfected with a plasmid containing CADM1 3'UTR and scrambled miRNAs, which were used as a negative control. In the experimental group, 293T cells were co-transfected with the plasmid containing CADM1 3'UTR and miR-214-3p mimic. The luciferase activity was measured using a dual-luciferase reporter assay. Each experiment was repeated $\geq 3$ times in triplicate. ${ }^{*} \mathrm{P}<0.05,{ }^{* *} \mathrm{P}<0.01$ vs. NC. miR, microRNA; 3'-UTR, 3'untranslated region; CADM1, cell adhesion molecule 1; NC, negative control; Rluc/Fluc, Renilla/firefly luciferase activity.

siNC-transfected cells (Fig. 4F), suggesting that the knockdown of CADM1 was able to activate the P44/42 signaling pathway, which subsequently affected the cellular functions that are modulated by the pathway.

\section{Discussion}

Previous studies have demonstrated that miR-214 functions either as an oncogene or a tumor suppressor in a number of human cancer types, including lung, prostate, colorectal and esophageal cancer (15-18). Previous studies have indicated that elevated miR-214-3p is associated with OS progression, but a limited number of studies have focused on the function and mechanism of miR-214-3p $(10,11,19,20)$. In a previous study by the authors, it was demonstrated that upregulated miR-214 expression was associated with aggressive clinicopathological features (tumor size, metastasis status and response to pre-operative chemotherapy) and poor prognosis of pediatric osteosarcoma (11). In the present study, it was demonstrated that miR-214-3p may act as an oncogene, where it promotes the proliferation, migration and invasion of OS cells. The knockdown of miR-214-3p was able to decrease the cell growth rate and mobility, which was consistent with the results of Xu and Wang (21).

To clarify the potential mechanism of miR-214-3p in OS, potential target genes were identified using target prediction tools. CADM1 was identified as a candidate target of miR-214-3p, which was verified by RT-qPCR and dual luciferase assays. 293T cells were co-transfected with CADM1 3'UTR-containing plasmids and scrambled miRNAs, which were used as a negative control. In the experimental group, 293T cells were co-transfected with CADM1 3'UTR-containing plasmids and miR-214-3p mimic. However, a plasmid containing a mutation in the seed sequence of the CADM1 3'UTR would provide a more convincing control for indicating the direct targeting of the CADM1 3'UTR by miR-214-3p.

CADM1 is located on chromosome 11q23.2, and is an intercellular adhesion molecule that is part of the immunoglobulin superfamily $(22,23)$. Silencing of CADM1 is frequently observed in various types of cancer, including lung, prostate, gastric, breast, pancreatic, nasopharyngeal and cervical cancer, and this is accompanied by increased proliferation, invasion and metastatic potential of tumors cell (24-27). However, to the best of our knowledge, only one previous study has 
A

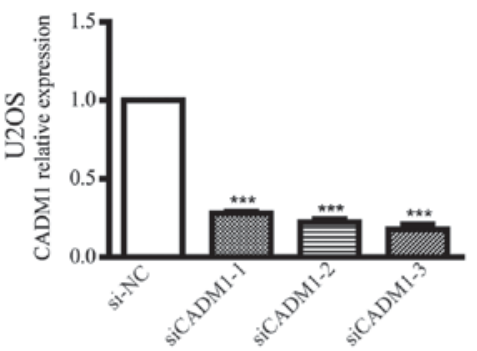

B

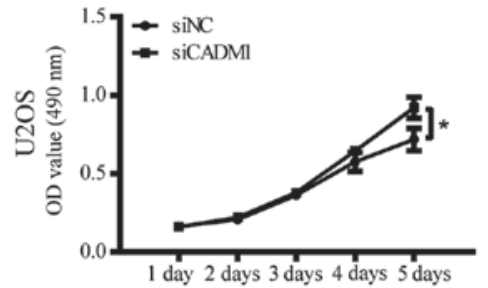

$\mathrm{C}$
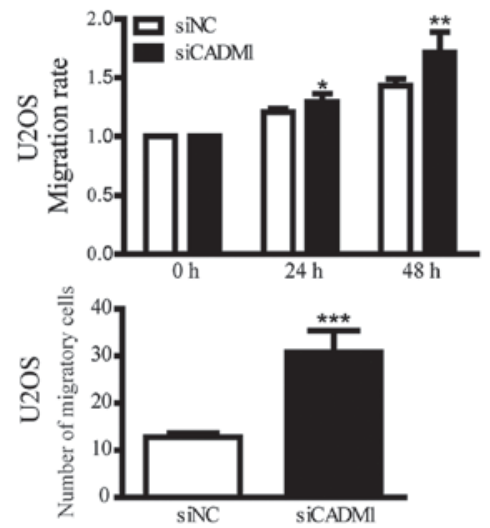

E

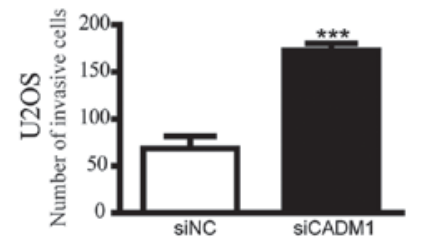

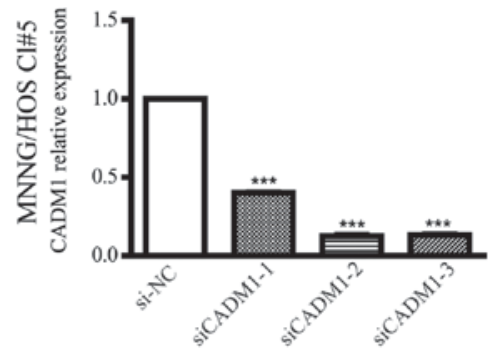
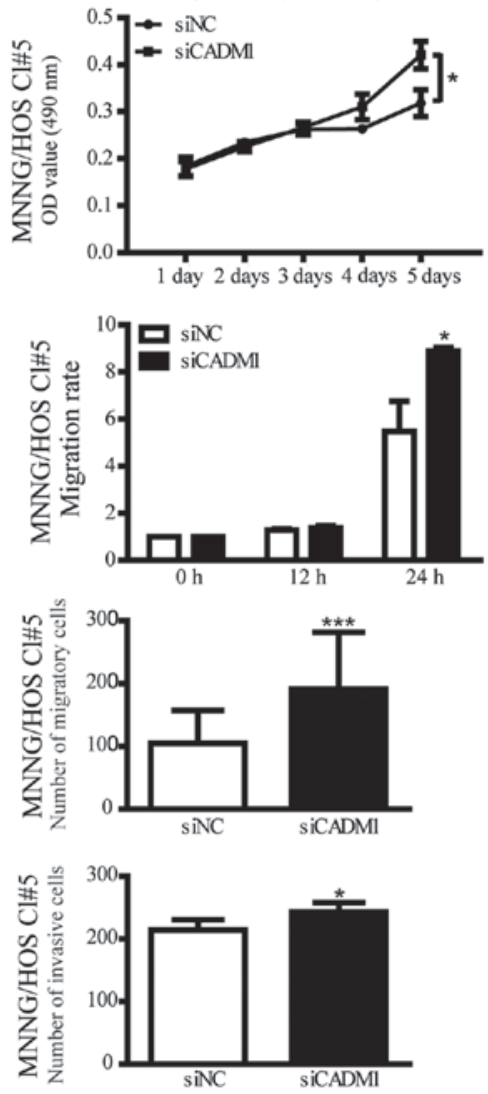

F

U2OS MNNG/HOS Cl\#5
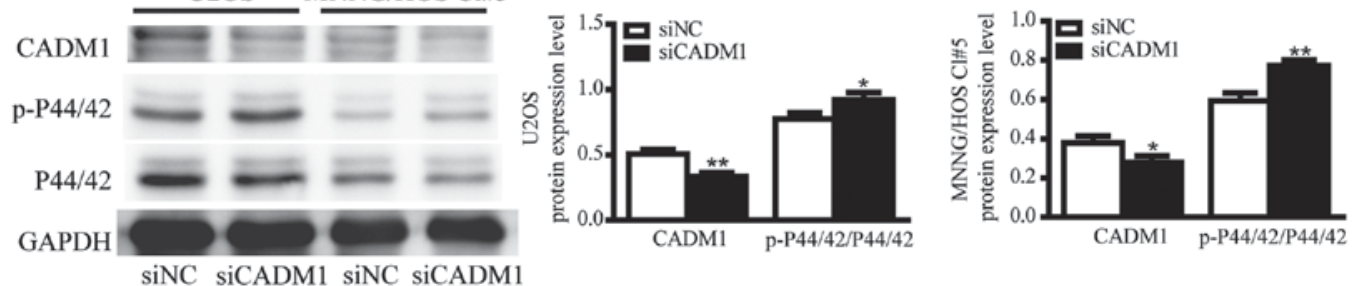

Figure 4. Knockdown of CADM1 promotes the proliferation, migration and invasion of OS cells by activating the extracellular-signal-regulated kinase signalling pathway. (A) Relative expression of CADM1 in U2OS and MNNG/HOS Cl\#5 cells that were transiently transfected with siCADM1, 2 or 3 or siNC. $18 \mathrm{~S}$ was used as an internal control. The value of each sample was calculated using the $2^{-\Delta \Delta C q}$ method and analyzed by one-way analysis of variance. Data are presented as the mean \pm standard deviation. (B) Growth curves of OS cells that were transfected with siCADM1 or siNC represent OD values at $490 \mathrm{~nm}$ measured by MTT assays. (C) A scratch assay was used to detect the motility of U2OS and MNNG/HOS Cl\#5 cells that were transfected with siCADM1 or siNC. (D) Quantitative results of Transwell migration assays in CADM1 knocked down-U2OS and MNNG/HOS Cl\#5 cells. U2OS and MNNG/HOS $\mathrm{Cl} \# 5$ cells were incubated for $24 \mathrm{~h}$. (E) Quantitative results of Transwell invasion assays in CADM1 knocked down-U2OS and MNNG/HOS Cl\#5 cells. U2OS and MNNG/HOS Cl\#5 cells were incubated for $48 \mathrm{~h}$. (F) The protein expression levels of CADM1, p-P44/42 MAPK and P44/42 MAPK were measured by western blotting. GAPDH was used as an internal control and Student's t-test was used. Data are presented as the mean \pm standard deviation. "P $<0.05$, ${ }^{* *} \mathrm{P}<0.01,{ }^{* * * *} \mathrm{P}<0.001$ vs. siNC. CADM1, cell adhesion molecule 1; si, small interfering RNA; NC, negative control; OD, optical density; OS, osteosarcoma; p-, phosphorylated; siNC, negative siRNA control.

investigated CADM1 in OS, which indicated that CADM1 may be a potential diagnostic marker (22). In the present study, CADM1 expression was suppressed using siRNA, and that downregulation of CADM1 resulted in increased proliferative, migratory and invasive abilities of OS cells, which was consistent with the effects of miR-214-3p.

Although it was demonstrated that miR-214-3p was able to modulate the proliferative, migratory and invasive abilities 
of OS cells by directly targeting the 3'UTR of CADM1, the molecular mechanism underlying the involvement of CADM1 remains unclear. CADM1 has been reported to be implicated in several pathways. Vallath et al (26) reported that CADM1 inhibited the progression of squamous cell carcinoma by reducing signal transducer and activator of transcription 3 activity. Zhang et al (24) demonstrated that CADM1 regulated the $\mathrm{G} 1 / \mathrm{S}$ phase transition and repressed tumorigenesis via the Rb-E2F pathway in hepatocellular carcinoma (24). Murakami et al (28) demonstrated that trans-homophilic interactions, mediated by CADM1, activated the phosphoinositide-3-kinase pathway to reorganize the actin cytoskeleton and form the epithelial cell structure. To the best of our knowledge, the present study is the first to demonstrate that the downregulation of CADM1 is able to activate the P44/42 MAPK signaling pathway, which has been reported to be associated with cell proliferation, migration and invasion $(29,30)$.

In conclusion, miR-214-3p was able to activate P44/42 MAPK signaling by downregulating CADM1 expression, thereby promoting the proliferation, migration and invasion of OS cells. These results indicate that miR-214-3p and CADM1 may be useful diagnostic markers for OS.

\section{Acknowledgements}

Not applicable.

\section{Funding}

The present study was supported by the Youth Project of Shanghai Municipal Commission of Health and Family Planning grant no. 20134y085) and the Natural Science Foundation of Shanghai (grant no. 14ZR1426400).

\section{Availability of data and materials}

All data generated or analyzed during this study are included in this published article.

\section{Authors' contributions}

ZGW made substantial contributions to conception and design, revised the manuscript critically for important intellectual content and gave final approval of the version to be published. HQC and MYM performed the literature research, experimental studies, data acquisition and data analysis. HQC wrote the manuscript.

\section{Ethics approval and consent to participate}

Not applicable.

\section{Consent for publication}

Not applicable.

\section{Competing interests}

The authors declare that there are no competing interests.

\section{References}

1. Ottaviani G and Jaffe N: The epidemiology of osteosarcoma. Cancer Treat Res 152: 3-13, 2009.

2. Yang J and Zhang W: New molecular insights into osteosarcoma targeted therapy. Curr Opin Oncol 25: 398-406, 2013.

3. Martin M, Geudens I, Bruyr J, Potente M, Bleuart A, Lebrun M, Simonis N, Deroanne C, Twizere JC, Soubeyran P, et al: PP2A regulatory subunit $\mathrm{B} \alpha$ controls endothelial contractility and vessel lumen integrity via regulation of HDAC7. EMBO J 32: 2491-2503, 2013

4. Kakimoto Y, Ito S, Abiru H, Kotani H, Ozeki M, Tamaki K and Tsuruyama T: Sorbin and SH3 domain-containing protein 2 is released from infarcted heart in the very early phase: Proteomic analysis of cardiac tissues from patients. J Am Heart Assoc 2: e000565, 2013.

5. Bielack SS, Hecker-Nolting S, Blattmann C and Kager L: Advances in the management of osteosarcoma. F1000Res 5: 2767, 2016.

6. Zhen N, Yang Q, Zheng K, Han Z, Sun F, Mei W and Yu Y: MiroRNA-127-3p targets XRCC3 to enhance the chemosensitivity of esophageal cancer cells to a novel phenanthroline-dione derivative. Int J Biochem Cell Biol 79: 158-167, 2016.

7. Inui M, Martello G and Piccolo S: MicroRNA control of signal transduction. Nat Rev Mol Cell Biol 11: 252-263, 2010.

8. Sharma T, Hamilton R and Mandal CC: miR-214: A potential biomarker and therapeutic for different cancers. Future Oncol 11: 349-363, 2015.

9. Penna E, Orso F and Taverna D: miR-214 as a key hub that controls cancer networks: Small player, multiple functions. J Invest Dermatol 135: 960-969, 2015.

10. Allen-Rhoades W, Kurenbekova L, Satterfield L, Parikh N, Fuja D, Shuck RL, Rainusso N, Trucco M, Barkauskas DA, Jo E, et al: Cross-species identification of a plasma microRNA signature for detection, therapeutic monitoring, and prognosis in osteosarcoma. Cancer Med 4: 977-988, 2015.

11. Wang Z, Cai H, Lin L, Tang M and Cai H: Upregulated expression of microRNA-214 is linked to tumor progression and adverse prognosis in pediatric osteosarcoma. Pediatr Blood Cancer 61: 206-210, 2014

12. Xu Y, He J, Wang Y, Zhu X, Pan Q, Xie Q and Sun F: miR-889 promotes proliferation of esophageal squamous cell carcinomas through DAB2IP. FEBS Lett 589: 1127-1135, 2015.

13. Livak KJ and Schmittgen TD: Analysis of relative gene expression data using real-time quantitative PCR and the 2(-Delta Delta C(T)) method. Methods 25: 402-408, 2001.

14. Liesenfeld M, Mosig S, Funke H, Jansen L, Runnebaum IB, Dürst M and Backsch C: SORBS2 and TLR3 induce premature senescence in primary human fibroblasts and keratinocytes. BMC Cancer 13: 507, 2013.

15. Zhao X, Lu C, Chu W, Zhang Y, Zhang B, Zeng Q, Wang R, Li Z, Lv B and Liu J: microRNA-214 governs lung cancer growth and metastasis by targeting carboxypeptidase-D. DNA Cell Biol 35: 715-721, 2016.

16. Srivastava A, Goldberger H, Dimtchev A, Ramalinga M, Chijioke J, Marian C, Oermann EK, Uhm S, Kim JS, Chen LN, et al: MicroRNA profiling in prostate cancer-the diagnostic potential of urinary miR-205 and miR-214. PLoS One 8: e76994, 2013.

17. Cristobal I, Caramés C, Madoz-Gurpide J, Rojo F, Aguilera O and Garcia-Foncillas J: Downregulation of miR-214 is specific of liver metastasis in colorectal cancer and could play a role determining the metastatic niche. Int J Colorectal Dis 29: 885, 2014.

18. Lu Q, Xu L, Li C, Yuan Y, Huang S and Chen H: miR-214 inhibits invasion and migration via downregulating GALNT7 in esophageal squamous cell cancer. Tumour Biol 37: 14605-14614, 2016.

19. Teicher BA: Searching for molecular targets in sarcoma. Biochem Pharmacol 84: 1-10, 2012.

20. Poos K, Smida J, Nathrath M, Maugg D, Baumhoer D and Korsching E: How microRNA and transcription factor co-regulatory networks affect osteosarcoma cell proliferation. PLoS Comput Biol 9: e1003210, 2013.

21. Xu Z and Wang T: miR-214 promotes the proliferation and invasion of osteosarcoma cells through direct suppression of LZTS1. Biochem Biophys Res Commun 449: 190-195, 2014.

22. Inoue T, Hagiyama M,Enoki E, Sakurai MA, Tan A, Wakayama T, Iseki S, Murakami Y, Fukuda K, Hamanishi C and Ito A: Cell adhesion molecule 1 is a new osteoblastic cell adhesion molecule and a diagnostic marker for osteosarcoma. Life Sci 92: 91-99, 2013. 
23. You Y, Zhang J, Li Y, Li Y, Shi G, Ma L and Wei $\mathrm{H}$ : CADM1/TSLC1 inhibits melanoma cell line A375 invasion through the suppression of matrix metalloproteinases. Mol Med Rep 10: 2621-2626, 2014.

24. Zhang W, Xie HY, Ding SM, Xing CY, Chen A, Lai MC, Zhou L and Zheng SS: CADM1 regulates the G1/S transition and represses tumorigenicity through the Rb-E2F pathway in hepatocellular carcinoma. Hepatobiliary Pancreat Dis Int 15: 289-296, 2016.

25. Yang Z, Wang R, Zhang T and Dong X: MicroRNA-126 regulates migration and invasion of gastric cancer by targeting CADM1. Int J Clin Exp Pathol 8: 8869-8880, 2015.

26. Vallath S, Sage EK, Kolluri KK, Lourenco SN, Teixeira VS, Chimalapati S, George PJ, Janes SM and Giangreco A: CADM1 inhibits squamous cell carcinoma progression by reducing STAT3 activity. Sci Rep 6: 24006, 2016.
27. Katoh M: Cardio-miRNAs and onco-miRNAs: Circulating miRNA-based diagnostics for non-cancerous and cancerous diseases. Front Cell Dev Biol 2: 61, 2014.

28. Murakami S, Sakurai-Yageta M, Maruyama T and Murakami Y: Trans-homophilic interaction of CADM1 activates PI3K by forming a complex with MAGuK-family proteins MPP3 and Dlg. PLoS One 9: e110062, 2014.

29. Cao Q, Mao ZD, Shi YJ, Chen Y, Sun Y, Zhang Q, Song L and Peng LP: MicroRNA-7 inhibits cell proliferation, migration and invasion in human non-small cell lung cancer cells by targeting FAK through ERK/MAPK signaling pathway. Oncotarget 7: 77468-77481, 2016.

30. Zhang Y, Wang J, Lv Z, Zhao D and Luo M: Cox-2 promotes mesenchymal stem cells differentiation into cardiocytes by activating JNK and ERK pathway. Biochem Biophys Res Commun 480: 101-105, 2016. 